\title{
Telomerase inhibitor imetelstat has preclinical activity across the spectrum of non-small cell lung cancer oncogenotypes in a telomere length dependent manner
}

\author{
Robin E. Frink ${ }^{1}$, Michael Peyton ${ }^{1}$, Joan H. Schiller ${ }^{2,4,8}$, Adi F. Gazdar ${ }^{1,3,4}$, Jerry W. \\ Shay $^{4,5,6}$ and John D. Minna ${ }^{1,4,7,8}$ \\ ${ }^{1}$ Hamon Center for Therapeutic Oncology Research, University of Texas Southwestern Medical Center, Dallas, TX, USA \\ 2 Inova Schar Cancer Institute, Falls Church, VA, USA \\ ${ }^{3}$ Department of Pathology, University of Texas Southwestern Medical Center, Dallas, TX, USA \\ ${ }^{4}$ Simmons Cancer Center, University of Texas Southwestern Medical Center, Dallas, TX, USA \\ ${ }^{5}$ Department of Cell Biology, University of Texas Southwestern Medical Center, Dallas, TX, USA \\ ${ }^{6}$ Center for Excellence in Genomics Medicine Research, King Abdulaziz University, Jeddah, Saudi Arabia \\ 7 Department of Pharmacology, University of Texas Southwestern Medical Center, Dallas, TX, USA \\ ${ }^{8}$ Department of Internal Medicine, University of Texas Southwestern Medical Center, Dallas, TX, USA \\ Correspondence to: John D. Minna, e-mail: John.Minna@utsouthwestern.edu \\ Keywords: imetelstat, telomerase, telomeres, lung cancer, telomerase inhibition \\ Received: December 04, 2015 Accepted: April 27, $2016 \quad$ Published: May 12, 2016
}

\section{ABSTRACT}

Telomerase was evaluated as a therapeutic oncotarget by studying the efficacy of the telomerase inhibitor imetelstat in non-small cell lung cancer (NSCLC) cell lines to determine the range of response phenotypes and identify potential biomarkers of response. A panel of 63 NSCLC cell lines was studied for telomere length and imetelstat efficacy in inhibiting colony formation and no correlation was found with patient characteristics, tumor histology, and oncogenotypes. While there was no overall correlation between imetelstat efficacy with initial telomere length (ranging from 1.5 to $20 \mathrm{~kb}$ ), the quartile of NSCLC lines with the shortest telomeres was more sensitive than the quartile with the longest telomeres. Continuous long-term treatment with imetelstat resulted in sustained telomerase inhibition, progressive telomere shortening and eventual growth inhibition in a telomere-length dependent manner. Cessation of imetelstat therapy before growth inhibition was followed by telomere regrowth. Likewise, in vivo imetelstat treatment caused tumor xenograft growth inhibition in a telomere-length dependent manner. We conclude from these preclinical studies of telomerase as an oncotarget tested by imetelstat response that imetelstat has efficacy across the entire oncogenotype spectrum of NSCLC, continuous therapy is necessary to prevent telomere regrowth, and short telomeres appears to be the best treatment biomarker.

\section{INTRODUCTION}

Lung cancer is the leading cause of cancer-related death in both men and women in the United States [1]. Non-small cell lung cancer (NSCLC) makes up $85 \%$ of all lung cancer cases and can be further categorized as adenocarcinoma, squamous cell carcinoma and large cell carcinoma. Standard "doublet" first line chemotherapies include a combination of paclitaxel or docetaxel, pemetrexed or gemcitabine, and carboplatin or cisplatin with tumor response rates of approximately $20-30 \%$. The recent advent of targeted agents such as erlotinib and crizotinib has yielded dramatic short-term clinical responses in patients with specific oncogene mutations (e.g. mutant EGFR or EML4-ALK fusions) showing the benefit of developing molecular analyses of tumors to 
implement "precision medicine" for lung cancer [2-4]. However, such approaches target only small subsets of patients with specific mutations and do not give curative clinical responses. Thus, new targeted therapeutic agents are needed, particularly for targets that are relevant to a large majority of lung cancers.

In this regard, telomerase is expressed in $85-90 \%$ of all cancers including lung cancer and is responsible for the limitless replication potential of cancer $[5,6]$. By contrast, telomerase is not expressed in most normal somatic cells making it an attractive, almost universal, target for cancer therapy. Telomeres, located at the ends of chromosomes, erode with every cell division due to the end replication problem, eventually leading to cellular senescence when telomeres become critically short [7]. When telomerase is active, telomere ends are re-elongated or stabilized allowing for continued cell divisions. The two functional components of telomerase are hTERT and hTR (hTERC). hTERT is a cellular reverse transcriptase that extends the ends of telomeres. hTR is a functional RNA that contains a telomere template component used by the reverse transcriptase to add the appropriate TTAGGG telomeric repeats to human telomeres during each replication cycle to counterbalance the losses due to the end replication problem [8-10]. Precancerous cells typically have very short telomeres and need to bypass senescence and crisis to continue cell proliferation, and therefore almost $90 \%$ of cancer cells have short telomeres relative to normal cells $[11,12]$. A subset of precancerous cells must turn on telomerase (or some alternative telomere lengthening mechanism) to become capable of extended growth potential. In addition, cancer stem cells (cancer initiating cells) need telomerase for their continued function [13]. Thus, targeted inhibition of telomerase in cancer cells should lead to critically short telomeres and subsequent senescence or cell death in tumor cells while causing minimal side effects on non-cancerous cells and could also be combined with chemotherapy or targeted therapy [13].

Imetelstat (formerly GRN163L) is a telomerase inhibitor that binds the hTR RNA template component of telomerase preventing telomerase from elongating telomeres. Imetelstat is a synthetic lipid-conjugated 13mer N3'P5' thio-phosphoramidate [14] (Supplemental Figure S1). Previous preclinical studies have shown efficacy of imetelstat in breast, prostate, liver, brain, pancreatic and bladder cancers as well as multiple myeloma and lymphoma [15-23]. Imetelstat has also shown efficacy in the A549 lung cancer cell line, but no additional preclinical work has been done to demonstrate imetelstat effectiveness in other NSCLCs [24]. Imetelstat has been tested in 14 clinical trials including a completed Phase I in NSCLC which determined safety and maximum tolerated dose in combination with paclitaxel and carboplatin chemotherapy as well as a randomized Phase II which used imetelstat as maintenance therapy for NSCLC [25]. What is not known is the efficacy of telomerase as an oncotarget in terms of the range of response to imetelstat in NSCLC preclinical models, whether there are correlations of imetelstat response with clinical characteristics, oncogenotypes, response to standard chemotherapy and targeted therapy agents, and whether imetelstat growth inhibition is associated with any tumor molecular biomarkers to facilitate a "precision medicine" based approach in future clinical trials.

In the present study, we looked at telomere length and growth responses to imetelstat in a large panel of NSCLC cell lines representing many different oncogenotypes as well as a large spectrum of standard chemotherapy and targeted therapy response phenotypes. We conclude that: imetelstat has activity (critically shortens telomeres, inhibits clonogenicity, and induces senescence) across the oncogenotype and standard chemotherapy/targeted therapy spectrum of NSCLCs; imetelstat response phenotypes correlate with tumor telomere length and treatment duration; and surprisingly, we found no evidence of acquired resistance with longterm therapy. These results validate telomerase as a relevant target and suggest that selecting patients whose tumors have the shortest quartile of telomeres may serve as an enrollment biomarker for telomerase inhibitors.

\section{RESULTS}

\section{NSCLCs exhibit heterogeneity of telomere length}

To better understand the telomere landscape of NSCLC, we used a panel of 63 NSCLC cell lines derived from patients with varying histology, stage, race, age, gender and smoking status (from here on referred to as "patient characteristics") and oncogenotypes (summarized in Supplemental Table S1 along with important, previously unpublished data on telomere length, colony forming efficacy, doubling times, and percent inhibition of colony formation by imetelstat). Average telomere length for each cell line was measured by TRF (telomere restriction fragment) via Southern blot and ranged from $1.5 \mathrm{~kb}$ to 20 $\mathrm{kb}$ (Figure 1). $75 \%$ of the lines had average telomeres of $5.5 \mathrm{~kb}$ or less and only 6 had average telomeres longer than $10 \mathrm{~kb}$. Thus, telomeres in most of these cancer cell lines are considerably shorter compared to normal (noncancerous) adult cells (7-15 kb).

Cell lines were divided into quartiles based on telomere length (Supplemental Figure S2A). The first quartile (Q1) average telomere length is $10.8 \mathrm{~kb}$ and is significantly longer than the three remaining quartiles of average $4.5 \mathrm{~kb}, 3.2 \mathrm{~kb}$, and $2.3 \mathrm{~kb}$ for quartile $2(\mathrm{Q} 2)$, quartile $3(\mathrm{Q} 3)$, and quartile 4 (Q4), respectively. There was no correlation between telomere length and patient characteristics or oncogenotype. From the longest quartile (Q1) to the shortest quartile (Q4), average smoking pack 
years is $58,45,45$ and 46, respectively, and average patient age is $55,54,52$ and 48 years old. The age range of patients from which the cell lines were derived was 25 to 73 years old, and overall there was no correlation between age of the patient and telomere length of the NSCLC line.
Imetelstat inhibits colony forming ability in a telomere length dependent manner across the entire spectrum of NSCLC oncogenotypes

The efficacy of imetelstat across the panel of NSCLC cell lines was assessed in a colony formation assay. Preliminary experiments determined $3 \mu \mathrm{M}$

A

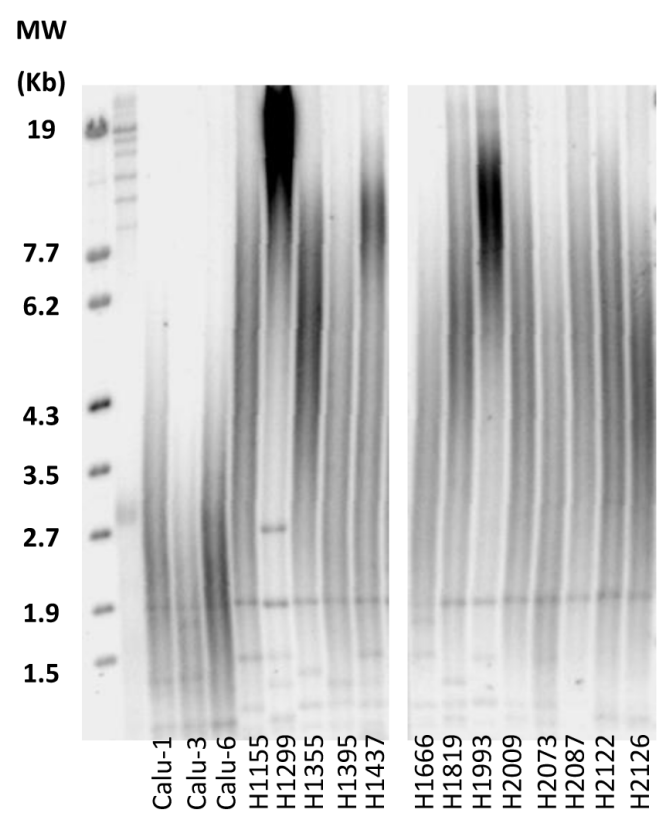

B

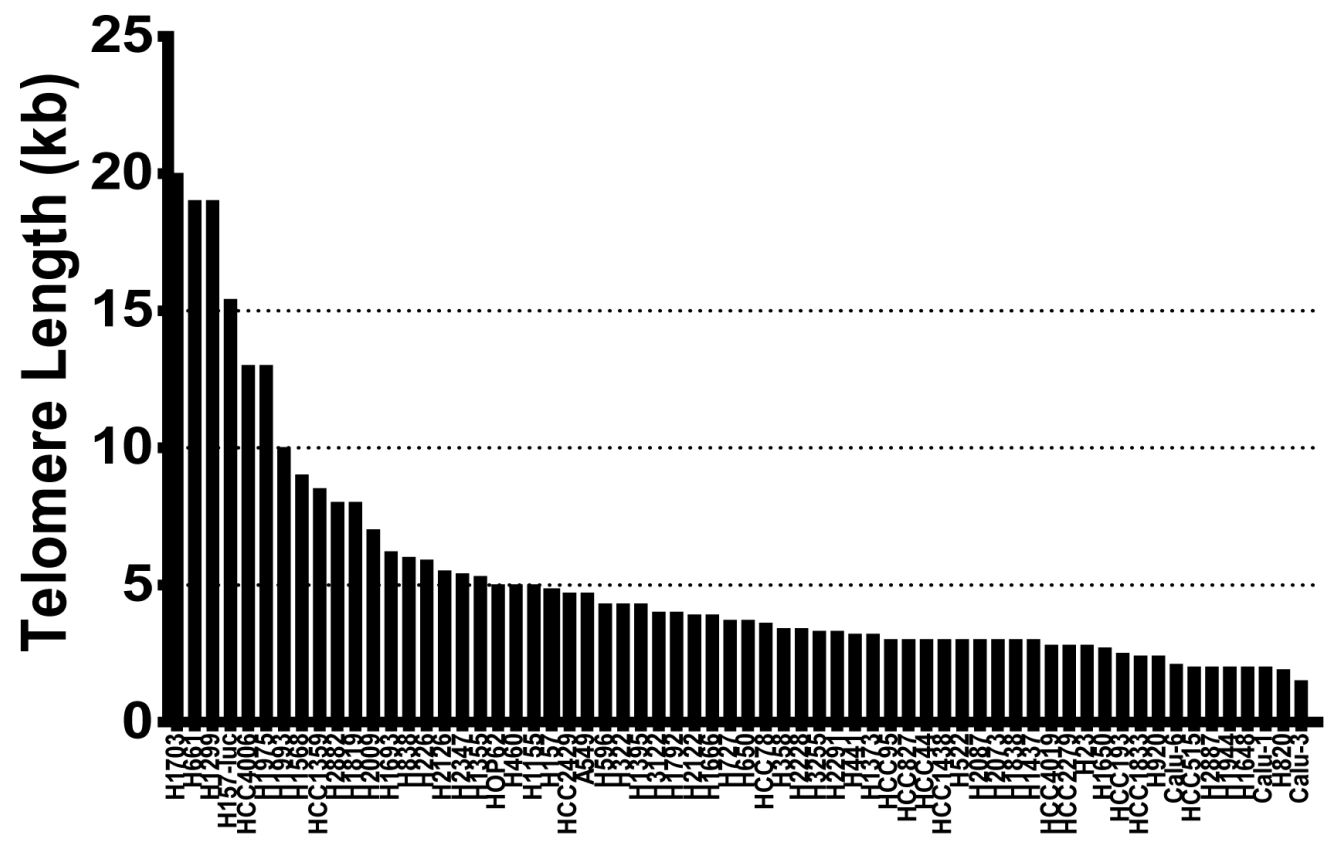

Figure 1: Heterogeneity of telomere length in NSCLC. A. Representative selection of telomere length variation in NSCLC cell lines via Southern blot. B. NSCLC panel average telomere length as measured by TRF (values listed in Supplemental Table S1). The names of the cell lines generated at NIH are officially NCI-Hxxxx and here abbreviated as Hxxxx. The lines generated at the University of Texas Southwestern Medical Center Hamon Center are designated HCCxxxx. Cell lines from other sources use the names given elsewhere. 
imetelstat was the optimal screening dose (Figure 2A2B). In all cases when colonies were harvested and pooled at the end of the colony formation assays, a onetime treatment with $3 \mu \mathrm{M}$ imetelstat greatly inhibited telomerase activity (Figure 2B). Response of a screen of 63 NSCLC lines ranged from 96\% inhibition in colony forming ability to an $84 \%$ increase in colony forming ability in the presence of imetelstat (Figure 2C-2D). While the possibility of NSCLC colony growth stimulation by imetelstat was surprising, when the number of untreated colonies was compared to treated colonies for the most resistant NSCLC line, H1703, the difference in colony forming efficiency was not statistically significant ( $p$ $>0.05)$. By contrast, for HCC44, the most imetelstat sensitive line, the difference was highly significant ( $p$ $<0.0001)$. Thus, the response to imetelstat ranged from $96 \%$ inhibition in colony forming ability to no statistically significant response. The response phenotypes did not correlate with patient characteristics or oncogenotype. When response of the NSCLC lines to imetelstat colony
A

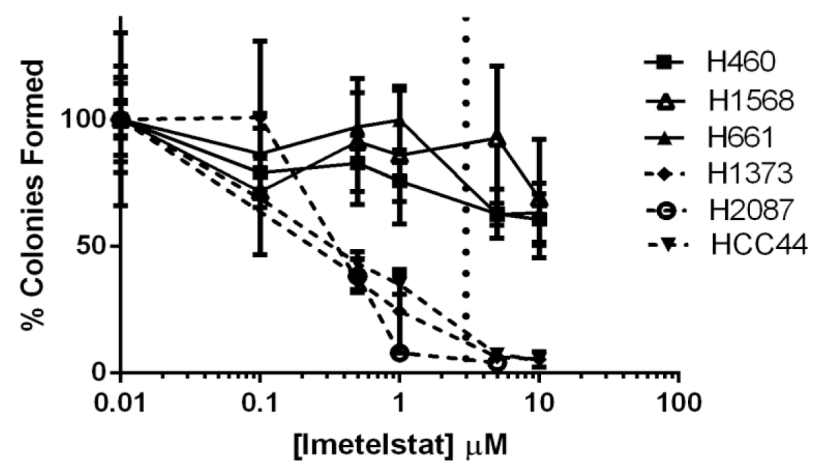

B

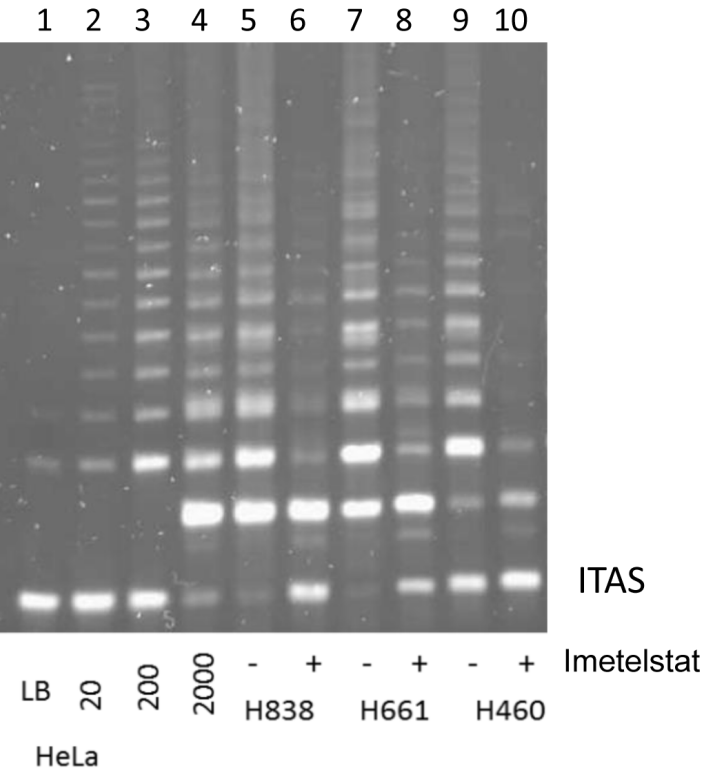

C

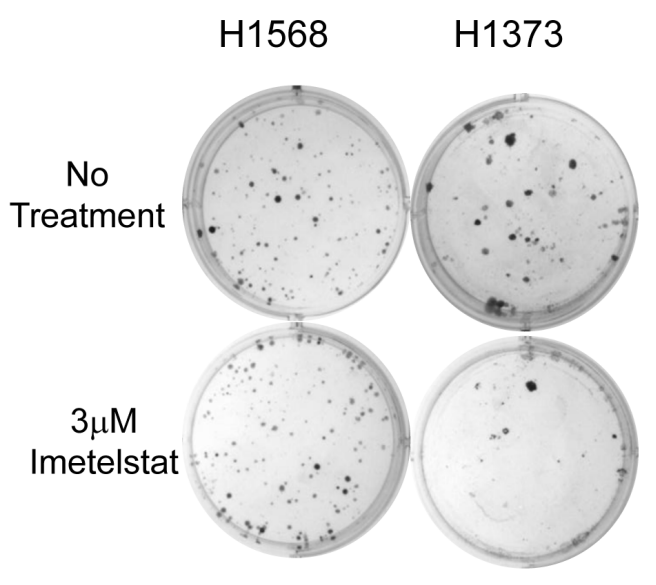

D

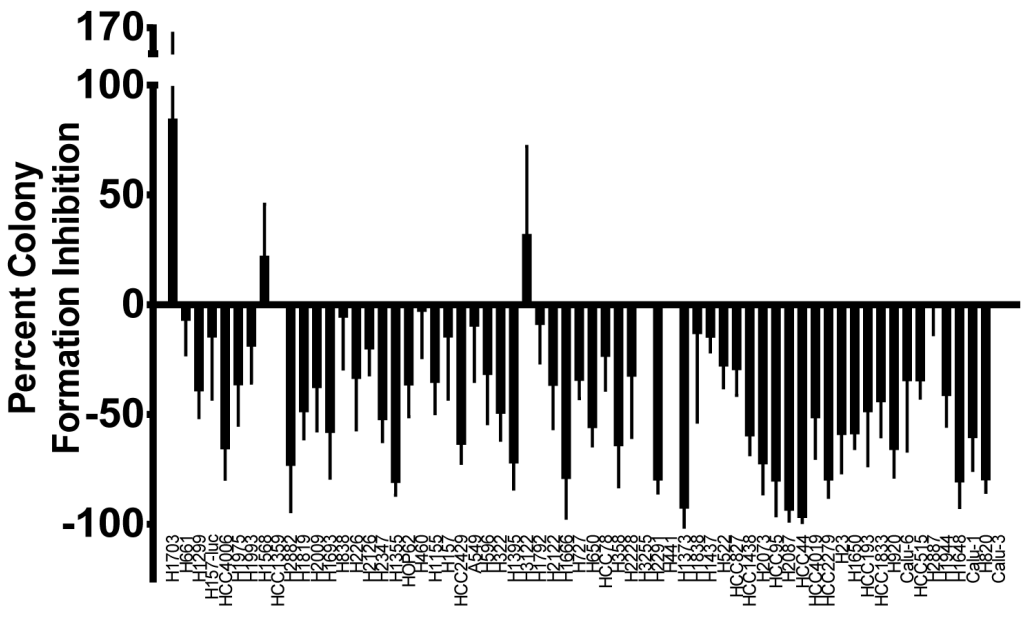

Figure 2: Range of responses to imetelstat treatment in NSCLC colony formation assay screen. A. Screen dose was determined by dose titrations of $0,0.1,0.5,1,5$, or $10 \mu \mathrm{M}$ imetelstat in colony formation. $3 \mu \mathrm{M}$ (the dashed vertical line) was chosen as the dose for the screen. B. TRAP assay to verify telomerase inhibition at the conclusion of colony formation assays. Control lanes 1-4, lysis buffer (LB) and 10, 200, 2000, HeLa cells. Lanes 5-6, $2000 \mathrm{H} 838$ cells from colony formation without and with $3 \mu \mathrm{M}$ imetelstat. Lanes 7-8 and 9-10 show H661 and H460, respectively, without and with imetelstat. ITAS: Internal Telomerase Assay Standard. C. Representative colony formation with no treatment versus $3 \mu \mathrm{M}$ imetelstat treatment. D. NSCLC cell line colony formation percent inhibition with $3 \mu \mathrm{M}$ imetelstat. Data represent average of at least 9 replicates; thin line is standard deviation. Values are given in Supplemental Table S1. For comparison, cell lines listed in same order of telomere length (Figure 1B). 
formation was compared to telomere length, an overall direct correlation was not observed (Supplemental Figure $\mathrm{S} 2 \mathrm{~B}$ ); however when telomere length quartiles were compared, cell lines with the shortest telomeres (Q4) were more sensitive to imetelstat compared to cell lines with the longest telomeres (Q1) (Supplemental Figure $\mathrm{S} 2 \mathrm{C}(p<0.03))$. Because there was minimal difference in telomere length between Q2, Q3 and Q4, these 3 quartiles were pooled and also show increased response compared to Q1 (Supplemental Figure S2C). While imetelstat response was correlated with colony forming efficiencies of untreated cells and doubling times, the correlation coefficients for these were very modest $\left(r^{2}\right.$ values of 0.18 and 0.12, respectively, Supplemental Figure S2D-E). The panel of NSCLC lines had legacy data on in vitro (MTS assay) response phenotypes for 28 standard and targeted chemotherapies and a range of sensitivity is observed with these drugs. There was no correlation between imetelstat response phenotypes and NSCLC response phenotypes to other therapies (Supplemental Table S2).

This panel of NSCLC cell lines contains "experiments of nature" - two NSCLC cell line pairs derived from the same tumor in each patient before and after neoadjuvant chemotherapy [26]. H1693 (diagnostic mediastinal lymph node biopsy) and H1819 (therapeutic tumor resection after neoadjuvant chemotherapy) were derived 6 months apart [26] and have average telomere lengths of 6.2 and $8 \mathrm{~kb}$, respectively, as well as similar imetelstat colony formation inhibition of $\sim 50 \%$. By contrast, H1993 (diagnostic mediastinal lymph node biopsy) and H2073 (therapeutic tumor resection after neoadjuvant chemotherapy) were derived 3 months apart [26]. They have different telomere lengths of $\sim 10$ and $3 \mathrm{~kb}$, for H1993 and H2073, respectively, and different imetelstat colony formation inhibition of $18 \%$ and $73 \%$. These two cell line pairs highlight the general trend seen with the panel as a whole that shorter telomeres predict higher imetelstat responsiveness.

\section{Long-term imetelstat treatment inhibits telomerase and shortens telomeres in multiple NSCLC cell lines}

To further explore the correlation of telomere length to imetelstat response, we examined long-term exposure to imetelstat in nine NSCLC cell lines (Calu-3, H157luc, H358, H460, H1648, H1819, H2087, H2887, and HCC827) which represent a range of telomere lengths, patient characteristics and oncogenotypes (Supplemental Table S1). $1 \mu \mathrm{M}$ imetelstat was given thrice weekly in mass culture based on the half-life estimates of imetelstat in tissue culture medium. Imetelstat treatment greatly reduced telomerase activity in H157-luc (initial telomere length $15 \mathrm{~kb}$ ) and $\mathrm{H} 2087$ (initial telomere length $3 \mathrm{~kb}$ ) after 8 weeks of continuous treatment (Figure $3 \mathrm{~A}$ ). Telomere length was measured beginning at 8 weeks and then again at 4-week intervals. Irrespective of initial telomere length, telomeres showed progressive shortening by 8 weeks and continued to shorten with prolonged treatment up to 20 weeks, shown in Figure 3B. Similar results were seen in the other cell lines (data not shown).

\section{Removal of imetelstat treatment reverses telomere shortening effects}

At 40 weeks of treatment, H157-luc cells were still growing in mass culture with no visible changes. To determine if telomere shortening was reversible, imetelstat therapy was stopped after 40 weeks of in vitro treatment ( $\sim 180$ population doublings) and cells were grown in the absence of drug. Cells were collected at 2, 4, 6 and 8 weeks after removal of imetelstat and telomere length assessed (Figure 3C). At 40 weeks of imetelstat treatment, telomere length was shortened to $2.6 \mathrm{~kb}$, almost $12 \mathrm{~kb}$ shorter compared to the untreated parental cells. After 2 weeks or $\sim 12$ population doublings without treatment, telomeres lengthen to $3.4 \mathrm{~kb}$. At 4 weeks ( $\sim 27$ population doublings) after removal of treatment, some H157-luc telomeres returned to parental length and all returned to parental length and remained there after 6 weeks (39 population doublings) in the absence of imetelstat.

\section{Long-term imetelstat treatment results in eventual} senescence and reduced colony forming ability

Continuous in vitro treatment of cells in mass culture with imetelstat leads to telomerase inhibition and telomere shortening regardless of initial telomere length, however the necessary treatment time to achieve senescence is dependent on the initial telomere length. Calu-3 had the shortest initial telomeres in the panel $(1.5 \mathrm{~kb})$ and the shortest response-time to continuous imetelstat treatment. The growth rate slowed in less than 4 population doublings (11 days) and loss of replicative potential and concomitant decrease in the number of cells followed with continued treatment (Figure 4A). H1648 had the next shortest initial telomere length $(2 \mathrm{~kb})$ and responded with slowed growth rate in 15 population doublings at $\sim 2$ months of treatment (Figure 4B). H2887, HCC827 and H460 had initial telomere lengths of $2 \mathrm{~kb}$ to $5 \mathrm{~kb}$ but required $\sim 12$ population doublings ( $\sim 25$ days), 40 population doublings ( $\sim 100$ days) and 50 population doublings ( $\sim 70$ days) for growth inhibition, respectively (Figure 4C-4E). An increase in senescence-associated $\beta$-galactosidase staining in long-term treated cells compared to parental indicates the slowed growth rate was due to an increase in senescing cells (Supplemental Figure S3).

The effect of shortened telomeres on colony forming ability was tested at multiple time points throughout the long-term treatment. NSCLC H1648 colony forming ability decreased after 56 days of treatment and continued to decrease up to 91 days of treatment (Figure 4F). NSCLC H460, which had longer initial telomeres and required longer imetelstat exposure for cells to senesce, showed minimal difference in colony forming ability after 56 days of treatment. However, by 91 days of treatment, there was a significant reduction in colony forming ability 

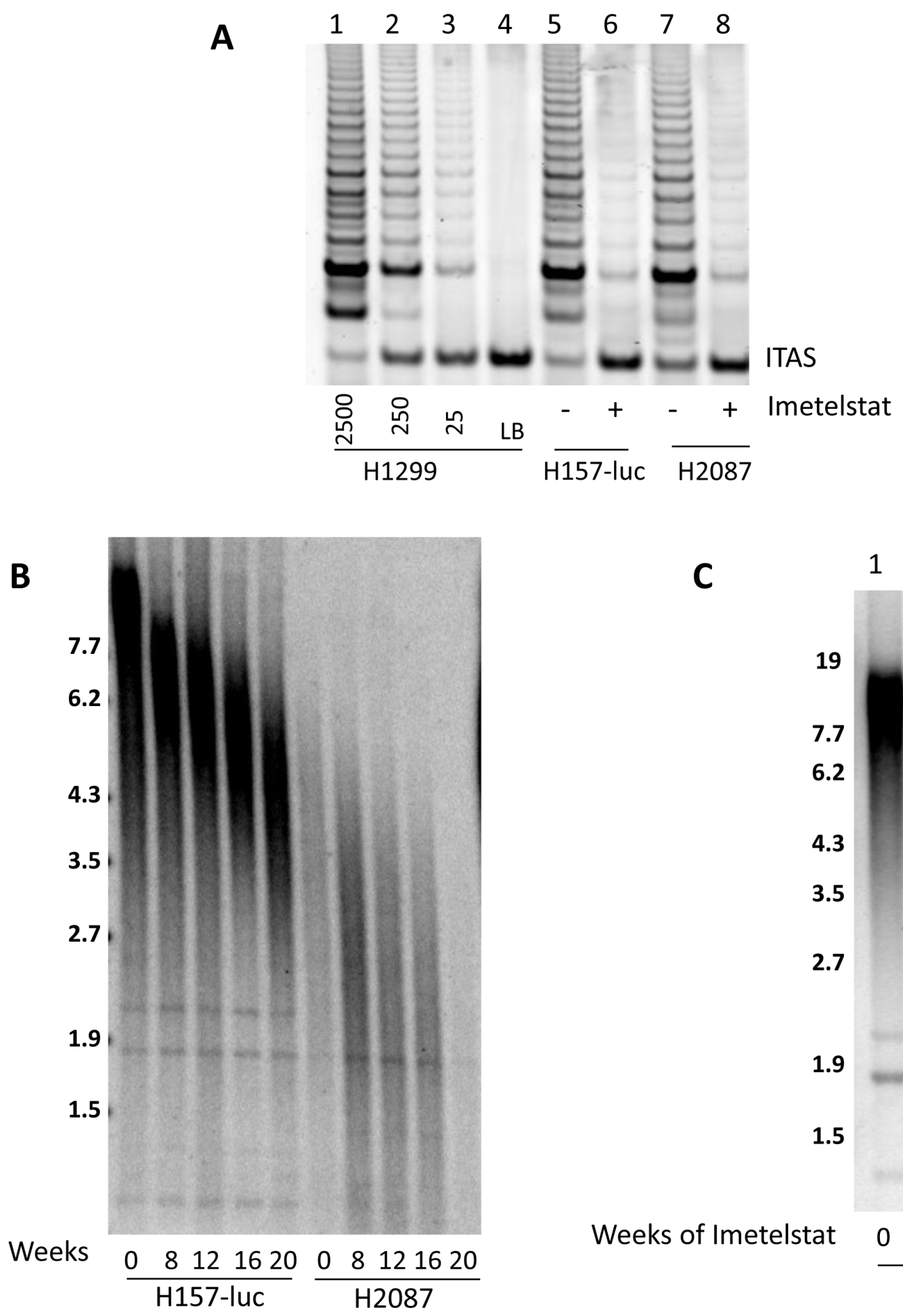

C

19

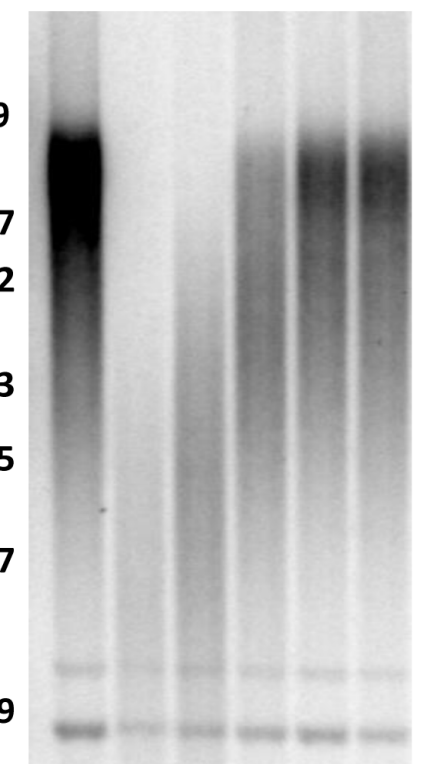

1.5

Weeks of Imetelstat

$\frac{0 \quad 40-2 \quad-4 \quad-6 \quad-8}{\text { H157-luc }}$

Figure 3: Imetelstat inhibits telomerase and leads to telomere shortening while removal of imetelstat results in regrowth of telomeres. NSCLC cell lines H157-luc and H2087 were treated with $1 \mu \mathrm{M}$ imetelstat. A. Telomerase activity after 8 weeks of treatment. Control lanes 1-4, 2500, 250, 25, H1299 cells and lysis buffer. Lanes 5-6, 2500 H157-luc cells and lanes 7-8 are H2087 cells without and with imetelstat for 8 weeks. ITAS: Internal Telomerase Assay Standard. B. H157-luc and H2087 parental telomere length and after $8,12,16$, and 20 weeks of $1 \mu \mathrm{M}$ imetelstat treatment. C. H157-luc telomere length after imetelstat removal. Lane 1 is parental telomere length of H157-luc and lane 2 is H157-luc after 40 weeks of $1 \mu \mathrm{M}$ imetelstat treatment at which point treatment ceased. Lanes 3-6 are H157-luc 2, 4, 6 and 8 weeks after removal of treatment. Note - H157-luc are NCI-H157 cells with an exogenous luciferase expression construct inserted. 
(Figure 4F). In both cell lines, as the telomeres shorten and growth slows, the ability of the cell line to form colonies decreased.

\section{Long-term imetelstat treatment of NSCLCs has a heterogeneous effect on response to standard chemotherapy and EGFR targeted therapies}

Throughout the long-term imetelstat treatment, the cell lines were tested periodically with standard chemotherapies to determine if imetelstat treatment and subsequent telomere shortening resulted in a change in response to other chemotherapies. Cell lines were treated in a 5-day cell viability assay with carboplatin, doxorubicin, gemcitabine, paclitaxel, paclitaxel/ carboplatin combination, erlotinib, and vinorelbine - all drugs that have been used in NSCLC treatment. The cell lines initially had a wide range in response to each therapy (Supplemental Table S3). While imetelstat treatment occasionally increased the response to cytotoxic
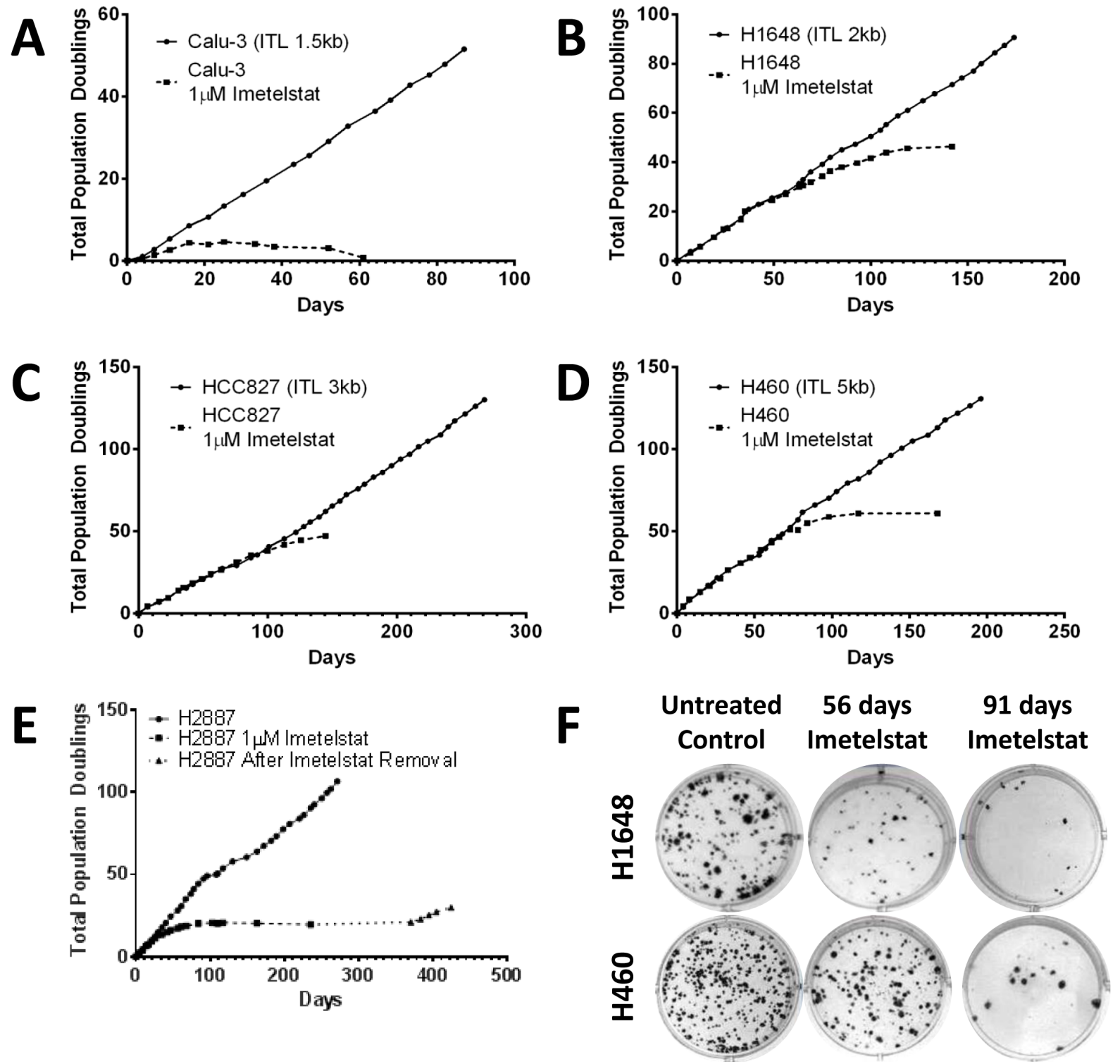

Figure 4: NSCLC growth and colony formation are inhibited after long-term imetelstat treatment. Cell lines were grown in the presence and absence of $1 \mu \mathrm{M}$ imetelstat given thrice weekly. Population doubling growth curves are shown for A. Calu-3, initial telomere length (ITL 1.5 kb), B. H1648 (ITL 2 kb), C. HCC827 (ITL 3 kb), D. H460 (ITL 5 kb), and E. H2887 (ITL 2 kb). In panel E note H2887 cells were treated long-term with imetelstat and growth slowed in 12 population doublings. At 235 days, imetelstat was removed. Dotted line with triangle indicates growth without imetelstat. F. H1648 and H460 liquid colony formation assays for untreated controls and for tumor cells treated with $1 \mu \mathrm{M}$ imetelstat for 56 and 91 days that demonstrate dramatic loss of colony forming ability after long-term imetelstat treatment. 
chemotherapy indicated by a change in $\mathrm{IC}_{50}$ values, this was highly heterogeneous between tumors and only occasionally gave dramatic decreases in IC50 values (see vinorelbine effect in 3/5 NSCLCs in Supplemental Table S3).

\section{Imetelstat slows tumor growth and results in telomere shortening in vivo in a telomere length dependent manner}

The in vivo efficacy of imetelstat was assessed using four cell lines (HCC827, Calu-3, H1648, and H460) in a subcutaneous xenograft model. Calu-3 tumor growth rate was significantly slower in the imetelstat treated group compared to control and final tumor weights were smaller $(p<0.0008$, Figure 5A). HCC827 and H1648 also showed slower growth rates and decreased tumor size in imetelstat treated tumors versus control tumors $(p<0.0008$ and $p<$ 0.007, respectively, Figure 5B-5C).

H460 had the longest telomeres tested in vivo and showed no difference in growth before tumors reached maximum tumor burden (Figure 5D), however there was a decrease in telomerase activity and telomere length in the treated tumors (Supplemental Figure S4A-B). H460 is also the fastest growing cell line tested in vivo reaching maximum tumor burden in about 3 weeks. To determine if shortened telomeres can reduce tumor forming ability, H460 was pretreated in vitro for 12 weeks before tumor cell injection. This pretreatment led to a delay in tumor formation compared to the untreated parental line (Supplemental Figure S4C). Therefore, imetelstat effectiveness in vivo is dependent on initial telomere length and treatment duration of imetelstat therapy.

\section{DISCUSSION}

In the present study, we analyzed the efficacy of the telomerase inhibitor imetelstat across a large panel of NSCLC cell lines including differing patient characteristics, oncogenotypes, and telomere lengths. NSCLCs varied $\sim 2$ logs in their imetelstat response phenotype in colony formation. Approximately 20\% of all NSCLC lines demonstrated dramatic inhibition to imetelstat in the colony formation assay, and this was seen over all NSCLC oncogenotypes. Telomere length ranged from $1.5 \mathrm{~kb}$ to $20 \mathrm{~kb}$, but $75 \%$ of the panel was less than $5.5 \mathrm{~kb}$ and this subset had the greatest response to imetelstat. NSCLC lines treated long-term (up to nine months) include adenocarcinoma, large cell and squamous cell carcinoma histology; Caucasian and African American patients; males and females ranging in age from 25 to 73 ; a range of oncogenotypes including different combinations of p53, KRAS, LKB1/STK11, and EGFR mutations (Supplemental Table S1), and both sensitive and resistant lines to standard chemotherapy and targeted therapies. Imetelstat effectively inhibited telomerase resulting in shortening of telomeres in all cell lines tested regardless of any clinical or genetic characteristics. This supports inhibition of telomerase as a universal cancer therapy target in telomerase expressing tumors. Time to senescence or cell death, however, was dependent on initial telomere length and growth rate of the cell line. These results were also seen in vivo where initial telomere length is proportional to the treatment time necessary to see significant differences in tumor growth rate.

The mechanism of action of imetelstat dictates prolonged treatment is necessary to observe progressive telomere shortening with continued cell division and eventual senescence or cell death. This is accompanied by a lag phase with minimal phenotypic change other than decreased telomere length and suggests shorter telomeres would have faster response times. As expected, initial telomere length correlated with the number of population doublings and time required to achieve critically short telomeres required to trigger these effects both in vitro and in vivo. Calu-3, H1648, and HCC827 with initial telomere lengths of $1.5 \mathrm{~kb}, 2 \mathrm{~kb}$, and $3 \mathrm{~kb}$, respectively, all demonstrated slowed growth in vitro in a length dependent manner and showed reduced tumor burden in the presence of imetelstat in a xenograft model. H460 (initial telomere length $5 \mathrm{~kb}$ ) required more population doublings to reach a slowed growth and eventual cell death in vitro and showed no initial difference in tumor growth rate in the presence of imetelstat in vivo. However, with pre-treatment in vitro, even this originally non-responsive cell line shows a delay in tumor formation emphasizing the need for adequate imetelstat exposure. Taken together, these data support short telomeres as selection criteria for maximum response to imetelstat in patients.

We potentially expected to see resistant subpopulations grow out during the extended culturing in vitro; surprisingly we saw no evidence for emerging resistance. To examine resistance in another way, we serial cloned H1648 in the presence of imetelstat, but once again saw no evidence of selection of resistant clones (Supplemental Figure S5). While these experiments are not definitive, they may speak to the unique biology of telomere extension using an RNA template and telomerase. Mutations in either of these required components, which might cause drug resistance, would in fact be predicted to give the same phenotype as the drug itself. Finally, one of the sensitive lines tested herein (H2073) expresses functional multi-drug resistance transporter (MDR) implying that this will not be a mechanism of resistance to imetelstat treatment.

Our results (Figure 3C and 4E) along with Burchett, et al [23] demonstrate the importance of continual treatment. After 40 weeks of treatment, telomeres are significantly reduced in length in H157-luc cells but telomeres regrew in as few as two weeks when imetelstat exposure was stopped. In $\mathrm{H} 2887$ cells, imetelstat was removed after 140 days of minimal growth/senescence and post removal of imetelstat, the cell line eventually 

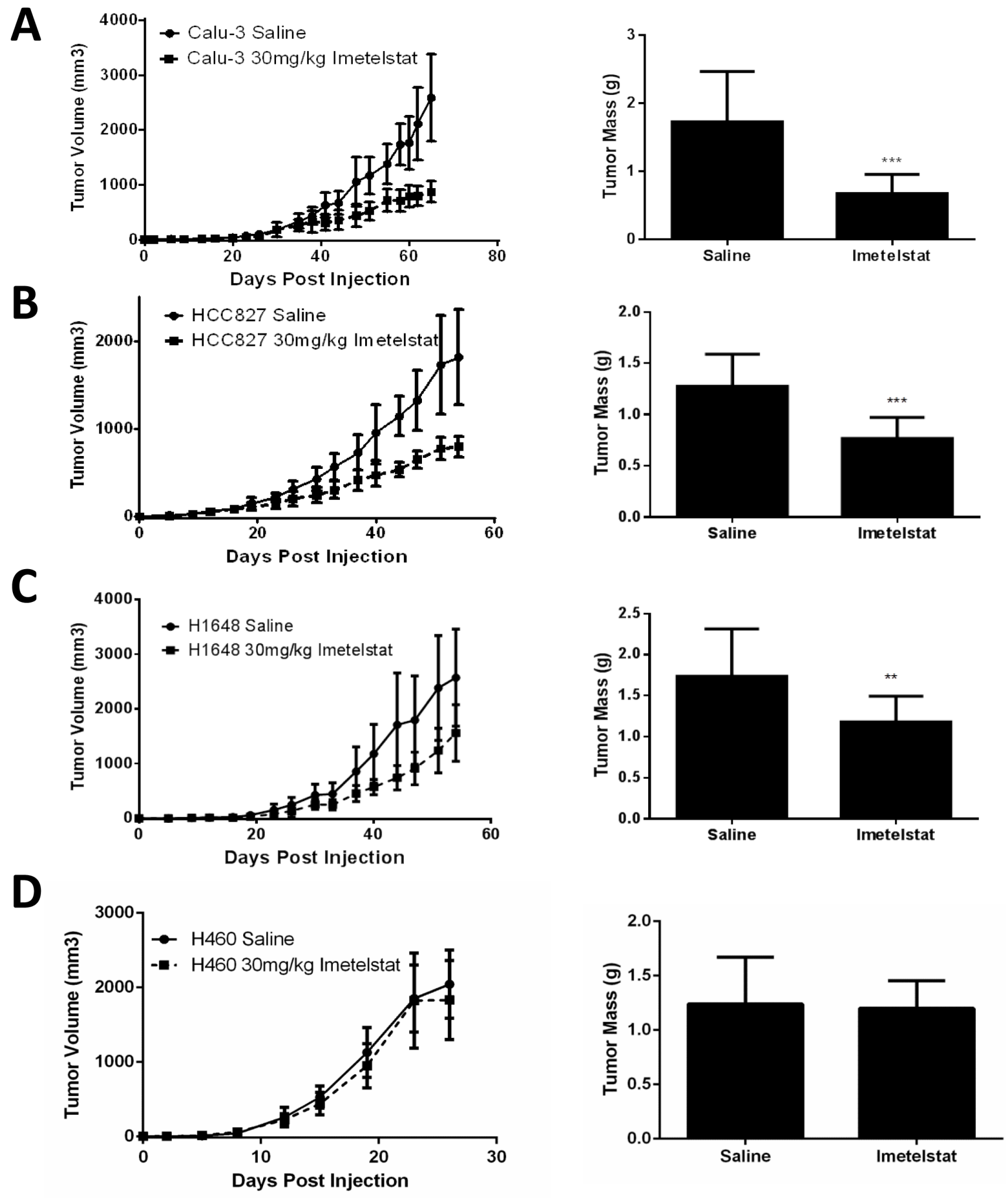

Figure 5: Imetelstat treatment in vivo reduces tumor burden. A. Calu-3 (initial telomere length (ITL) $1.5 \mathrm{~kb}$ ) tumor growth curves with $30 \mathrm{mg} / \mathrm{kg}$ imetelstat versus control and final tumor weights ( $n=10$ per group). B. HCC827 (ITL $3 \mathrm{~kb})$ tumor growth curves with imetelstat and final tumor weights ( $n=10$ per group). C. H1648 (ITL $2 \mathrm{~kb}$ ) tumor growth curves and final tumor weights $(n=12$ per group. D. H460 (ITL $5 \mathrm{~kb}$ ) tumor growth curves and final tumor weights. Error bars are standard deviation. $* * * p<0.0008, * * p<0.007$ as determined by student's $t$-test. 
recovers. Thus, once imetelstat treatment is begun and telomerase is inhibited, cells must be exposed to uninterrupted telomerase inhibition to ensure not only continued telomere shortening but also prevention of telomere re-elongation.

Although this study shows that all cell lines tested with imetelstat experienced a decrease in telomere length and concomitant loss of replicative potential, this study also indicates that given the therapeutic window, tumors with short telomeres will respond best to imetelstat therapy (Figure 5, Supplemental Figure S4). Indeed, the clinical trial of imetelstat in NSCLC provided information on a trend in which patients with the shortest telomeres responded best to therapy [25]. This study used imetelstat as maintenance therapy following standard chemotherapy. Due to the lag time necessary to achieve tumor cell death, imetelstat may work best in combination with first line therapy and continued as a maintenance therapy with other cytotoxic or targeted chemotherapies. In some cases long-term imetelstat can sensitize NSCLCs to cytotoxic therapies (Supplemental Table S3). Previous work has shown imetelstat can sensitize breast cancer to radiation $[16,27]$ or trastuzumab [28] and breast and liver cancer to doxorubicin $[16,19]$. While further studies will be required to determine the optimal combinations that are clinically relevant, some of our data supports the idea that imetelstat might best be used in combination with EGFR targeted therapy. For example, tumors with EGFR mutations making them susceptible to EGFR tyrosine kinase inhibitors (EGFR TKIs) have shown great initial response to EGFR TKIs in the clinic but frequently develop resistance [29]. HCC827, a NSCLC EGFR mutant line responsive to erlotinib [30], was effectively targeted with imetelstat and responded in a telomere length dependent manner both in vitro and in vivo. Imetelstat in combination with or following erlotinib treatment in EGFR mutant cancers would be predicted to work concomitantly by treating the EGFR mutant cancer cells with erlotinib while concurrently shortening telomeres. Cells that eventually become resistant to EGFR therapy would thus have short telomeres preventing their continued growth. Therefore, follow up studies should include combining imetelstat with targeted therapies such as erlotinib in EGFR mutant lung cancer and crizotinib in EML4-ALK fusion lung cancer. Additionally, with the advances of immunotherapeutic approaches to lung cancer that have recently developed, it will be important to determine if targeting telomerase also changes the immune response to lung cancer cells after various immunotherapies.

Our results, along with previous imetelstat studies, support continued investigation of telomerase inhibition in NSCLC, particularly for tumors with short telomeres. This could be with imetelstat or other telomerase targeting agents. Based on the NSCLC panel we studied, the subset of NSCLC potentially sensitive to telomerase-targeted therapy could be the large majority of all NSCLC patients.
We emphasize that imetelstat and thus telomerase-targeted therapy can be effective in NSCLCs with many different oncogenotypes and drug response phenotypes. In addition, imetelstat dosage schedules must ensure continuous treatment so drug induced telomere shortening is not negated by drug free intervals allowing telomere regrowth. In conclusion, the present results support continued testing of telomerase targeted therapy across the full spectrum of NSCLCs, with any early phase clinical trials directed at patients shown to have the shortest quartile of telomere length at the initiation of therapy to provide an enrollment enrichment biomarker for telomerase targeted cancer therapy.

\section{MATERIALS AND METHODS}

\section{Tumor cell lines and cell culture}

With the exception of Calu-1, Calu-3, Calu-6, A549 and HOP62 (obtained from ATCC), all other cell lines were established in the lab of Drs. John Minna and Adi Gazdar at the NIH (referred to as NCI-Hxxxx, or Hxxxx) or UT Southwestern Medical Center (referred to as HCCxxxx) (1981-2014) and are available through ATCC or the Hamon Center. Cells were cultured in RPMI with $5 \%$ fetal bovine serum in $5 \% \mathrm{CO}_{2}$ at $37^{\circ} \mathrm{C}$. $\mathrm{H} 157-$ luc expresses firefly luciferase which was established as previously described [24] and was used in some experiments because its telomere length is $15.4 \mathrm{~kb}$ compared to H157 parental line whose telomere length is $4.8 \mathrm{~kb}$. All cell lines were authenticated by DNA fingerprinting with the Promega StemElite ID system (Cat\# G9530) and confirmed mycoplasma free by e-Myco kit (Boca Scientific). Imetelstat (Geron Corp) treatment began 24 hours after seeding and fresh drug $(1 \mu \mathrm{M})$ in PBS was given 2-3 times per week for continuous treatment. Population doubling (PD) was calculated as $[(\log N(t)$ $-\log N(\mathrm{t} 0)) / \log 2]$ where $\mathrm{N}(\mathrm{t})$ is the number of cells at time of passage and $\mathrm{N}(\mathrm{t} 0)$ is the number of cells seeded at previous passage. Doubling times were calculated by dividing number of hours from seeding to harvest by the PD.

\section{Telomerase activity}

Telomerase activity was measured using the telomerase repeat amplification protocol (TRAP) assay via the TRAPeze Telomerase Detection Kit (Millipore). 100,000 cells were resuspended in $100 \mu 1$ 1x CHAPS lysis buffer. $1 \mu \mathrm{l}$ (1000 cells) was used for the PCR reaction. For tumor samples, $5 \mu \mathrm{g}$ of protein were used. 


\section{Telomere length}

Telomere length was determined using a Southern blot protocol as previously described [31]. Briefly, DNA was obtained using the DNeasy kit (Qiagen) in the QiaCube (Qiagen). $1 \mu \mathrm{g}$ DNA was digested with a mix of equal parts AluI (New England Biosciences), CfoI (Promega), HaeIII (New England Biosciences), HinfI (New England Biosciences), MspI (New England Biosciences), and RsaI (New England Biosciences) restriction enzymes. The digested DNA was run on $0.7 \%$ agarose gel. The gel was denatured, dried, neutralized and hybridized with a radioactive probe to the telomeric sequence, exposed to a phosphor screen and scanned with a phosphor Imager.

\section{Colony formation}

500 cells were plated in triplicate in 6-well plates. The assay was terminated at 14-21 days when at least $50 \%$ of colonies had 50 or more cells per colony in control wells. Cells were fixed and stained with $6 \%$ glutaraldehyde and $0.5 \%$ crystal violet solution [32]. Colonies were counted by eye and confirmed by imaging plates with the ChemiDoc XRS+ Imager (Bio-Rad) and using the Colony Counting function of Quantity One Software (v4.6.5, BioRad). Each assay was repeated a minimum of 3 times per cell line. For the panel screen, $3 \mu \mathrm{M}$ imetelstat was added 24 hours after seeding. Long-term treated cells did not receive imetelstat in the assay. Percent colony forming efficiency was calculated as (number of colonies counted/ number of cells seeded)*100.

\section{SA- $\beta$-gal staining}

Senescence associated $\beta$-galactosidase staining was performed as previously described [33].

\section{Tumor xenografts}

Mouse work followed an IACUC approved protocol. $1 \mathrm{x} 106$ cells were injected subcutaneously into the right flank of 6-8 week old female NOD/SCID mice. Mice were randomized with 10 mice per group and treatment began 1 day after cell injection. $30 \mathrm{mg} / \mathrm{kg}$ imetelstat in saline or saline alone was given in $100 \mu \mathrm{L}$ i.p. $3 x /$ week $[17,34,35]$. Tumors were measured using calipers to take perpendicular length measurements $2 \mathrm{x} /$ week. Tumor volumes were calculated as ( $\mathrm{L} \mathrm{x} \mathrm{w2)}(\pi / 6)$ where $\mathrm{L}$ is length and $\mathrm{w}$ is width, the shorter measurement. Mice were sacrificed when control tumors reached an average of $2000 \mathrm{~mm}^{3}$ when tumors were harvested and weighed. For the pre-treatment studies, $\mathrm{H} 460$ cells were treated with $1 \mu \mathrm{M}$ imetelstat for 12 weeks in vitro before injection and imetelstat treatment was not continued in vivo.

\section{ACKNOWLEDGMENTS}

We thank Ning Go and Katia Bassett from Geron Corp. who provided continual insight and feedback. We also thank Erin Kitten and Roswell Deutscher for their technical help, and Luc Girard for sharing chemotherapy response data and for help with data analysis and in-house software development.

\section{CONFLICTS OF INTEREST}

This work was partially supported by a research grant from Geron Corp.

\section{FUNDING}

This work was supported by NCI SPORE P50CA70907 (JD Minna, JH Schiller, JW Shay), P30CA142543 (JD Minna, JH Schiller, JW Shay), and CPRIT RP110708 (JD Minna) Geron Corp., Menlo Park, CA (RE Frink, JD Minna).

\section{REFERENCES}

1. Siegel R, Naishadham D and Jemal A. Cancer statistics, 2013. CA Cancer J Clin. 63:11-30.

2. Kris MG, Johnson BE, Berry LD, Kwiatkowski DJ, Iafrate AJ, Wistuba, II, Varella-Garcia M, Franklin WA, Aronson SL, Su PF, Shyr Y, Camidge DR, Sequist LV, et al. Using multiplexed assays of oncogenic drivers in lung cancers to select targeted drugs. JAMA. 2014; 311:1998-2006.

3. Sholl LM, Aisner DL, Varella-Garcia M, Berry LD, DiasSantagata D, Wistuba, II, Chen H, Fujimoto J, Kugler K, Franklin WA, Iafrate AJ, Ladanyi M, Kris MG, Johnson BE, Bunn PA, Minna JD, et al. Multi-institutional Oncogenic Driver Mutation Analysis in Lung Adenocarcinoma: The Lung Cancer Mutation Consortium Experience. Journal of thoracic oncology. 2015; 10:768-777.

4. Villaruz LC, Socinski MA, Abberbock S, Berry LD, Johnson BE, Kwiatkowski DJ, Iafrate AJ, Varella-Garcia M, Franklin WA, Camidge DR, Sequist LV, Haura EB, Ladanyi M, et al. Clinicopathologic features and outcomes of patients with lung adenocarcinomas harboring BRAF mutations in the Lung Cancer Mutation Consortium. Cancer. 2015; 121:448-456.

5. Kim NW, Piatyszek MA, Prowse KR, Harley CB, West MD, Ho PL, Coviello GM, Wright WE, Weinrich SL and Shay JW. Specific association of human telomerase activity with immortal cells and cancer. Science. 1994; 266:20112015.

6. Hiyama K, Hiyama E, Ishioka S, Yamakido M, Inai K, Gazdar AF, Piatyszek MA and Shay JW. Telomerase activity in small-cell and non-small-cell lung cancers. J Natl 
Cancer Inst. 1995; 87:895-902.

7. Wright WE and Shay JW. Historical claims and current interpretations of replicative aging. Nature biotechnology. 2002; 20:682-688.

8. Feng J, Funk WD, Wang SS, Weinrich SL, Avilion AA, Chiu CP, Adams RR, Chang E, Allsopp RC, Yu J and et al. The RNA component of human telomerase. Science. 1995; 269:1236-1241.

9. Harrington L, Zhou W, McPhail T, Oulton R, Yeung DS, Mar V, Bass MB and Robinson MO. Human telomerase contains evolutionarily conserved catalytic and structural subunits. Genes Dev. 1997; 11:3109-3115.

10. Meyerson M, Counter CM, Eaton EN, Ellisen LW, Steiner P, Caddle SD, Ziaugra L, Beijersbergen RL, Davidoff MJ, Liu Q, Bacchetti S, Haber DA and Weinberg RA. hEST2, the putative human telomerase catalytic subunit gene, is upregulated in tumor cells and during immortalization. Cell. 1997; 90:785-795.

11. Hastie ND, Dempster M, Dunlop MG, Thompson AM, Green DK and Allshire RC. Telomere reduction in human colorectal carcinoma and with ageing. Nature. 1990; 346:866-868.

12. Shay JW and Wright WE. Senescence and immortalization: role of telomeres and telomerase. Carcinogenesis. 2005; 26:867-874.

13. Buseman CM, Wright WE and Shay JW. Is telomerase a viable target in cancer? Mutation research. 2012; 730:9097.

14. Herbert BS, Gellert GC, Hochreiter A, Pongracz K, Wright WE, Zielinska D, Chin AC, Harley CB, Shay JW and Gryaznov SM. Lipid modification of GRN163, an N3'$>$ P5' thio-phosphoramidate oligonucleotide, enhances the potency of telomerase inhibition. Oncogene. 2005; 24:52625268.

15. Gellert GC, Dikmen ZG, Wright WE, Gryaznov S and Shay JW. Effects of a novel telomerase inhibitor, GRN163L, in human breast cancer. Breast Cancer Res Treat. 2006; 96:7381.

16. Hochreiter AE, Xiao H, Goldblatt EM, Gryaznov SM, Miller KD, Badve S, Sledge GW and Herbert BS. Telomerase template antagonist GRN163L disrupts telomere maintenance, tumor growth, and metastasis of breast cancer. Clin Cancer Res. 2006; 12:3184-3192.

17. Marian CO, Cho SK, McEllin BM, Maher EA, Hatanpaa KJ, Madden CJ, Mickey BE, Wright WE, Shay JW and Bachoo RM. The telomerase antagonist, imetelstat, efficiently targets glioblastoma tumor-initiating cells leading to decreased proliferation and tumor growth. Clin Cancer Res. 16:154-163.

18. Marian CO, Wright WE and Shay JW. The effects of telomerase inhibition on prostate tumor-initiating cells. International journal of cancer. 127:321-331.

19. Djojosubroto MW, Chin AC, Go N, Schaetzlein S, Manns
MP, Gryaznov S, Harley CB and Rudolph KL. Telomerase antagonists GRN163 and GRN163L inhibit tumor growth and increase chemosensitivity of human hepatoma. Hepatology. 2005; 42:1127-1136.

20. Dikmen ZG, Wright WE, Shay JW and Gryaznov SM. Telomerase targeted oligonucleotide thio-phosphoramidates in T24-luc bladder cancer cells. Journal of cellular biochemistry. 2008; 104:444-452.

21. Akiyama M, Hideshima T, Shammas MA, Hayashi T, Hamasaki M, Tai YT, Richardson P, Gryaznov S, Munshi $\mathrm{NC}$ and Anderson KC. Effects of oligonucleotide N3'-> P5' thio-phosphoramidate (GRN163) targeting telomerase RNA in human multiple myeloma cells. Cancer Res. 2003; 63:6187-6194.

22. Wang ES, Wu K, Chin AC, Chen-Kiang S, Pongracz K, Gryaznov S and Moore MA. Telomerase inhibition with an oligonucleotide telomerase template antagonist: in vitro and in vivo studies in multiple myeloma and lymphoma. Blood. 2004; 103:258-266.

23. Burchett KM, Yan Y and Ouellette MM. Telomerase inhibitor Imetelstat (GRN163L) limits the lifespan of human pancreatic cancer cells. PloS one. 2014; 9:e85155.

24. Dikmen ZG, Gellert GC, Jackson S, Gryaznov S, Tressler $\mathrm{R}$, Dogan P, Wright WE and Shay JW. In vivo inhibition of lung cancer by GRN163L: a novel human telomerase inhibitor. Cancer Res. 2005; 65:7866-7873.

25. Chiappori AA, Kolevska T, Spigel DR, Hager S, Rarick M, Gadgeel S, Blais N, Von Pawel J, Hart L, Reck M, Bassett E, Burington B and Schiller JH. A randomized phase II study of the telomerase inhibitor imetelstat as maintenance therapy for advanced non-small-cell lung cancer. Annals of oncology. 2015; 26:354-362.

26. Phelps RM, Johnson BE, Ihde DC, Gazdar AF, Carbone DP, McClintock PR, Linnoila RI, Matthews MJ, Bunn PA, Jr., Carney D, Minna JD and Mulshine JL. NCI-Navy Medical Oncology Branch cell line data base. Journal of cellular biochemistry Supplement. 1996; 24:32-91.

27. Gomez-Millan J, Goldblatt EM, Gryaznov SM, Mendonca MS and Herbert BS. Specific telomere dysfunction induced by GRN163L increases radiation sensitivity in breast cancer cells. Int J Radiat Oncol Biol Phys. 2007; 67:897-905.

28. Goldblatt EM, Erickson PA, Gentry ER, Gryaznov SM and Herbert BS. Lipid-conjugated telomerase template antagonists sensitize resistant HER2-positive breast cancer cells to trastuzumab. Breast Cancer Res Treat. 2009; 118:21-32.

29. Kosaka T, Yamaki E, Mogi A and Kuwano H. Mechanisms of resistance to EGFR TKIs and development of a new generation of drugs in non-small-cell lung cancer. J Biomed Biotechnol. 2011:165214.

30. Zhang Z, Lee JC, Lin L, Olivas V, Au V, LaFramboise T, Abdel-Rahman M, Wang X, Levine AD, Rho JK, Choi YJ, Choi CM, Kim SW, Jang SJ, Park YS, Kim WS, et al. 
Activation of the AXL kinase causes resistance to EGFRtargeted therapy in lung cancer. Nature genetics. 44:852860.

31. Herbert BS, Shay JW and Wright WE. Analysis of telomeres and telomerase. Curr Protoc Cell Biol. 2003; Chapter 18:Unit 1816.

32. Franken NA, Rodermond HM, Stap J, Haveman J and van Bree C. Clonogenic assay of cells in vitro. Nat Protoc. 2006; 1:2315-2319.

33. Debacq-Chainiaux F, Erusalimsky JD, Campisi J and Toussaint O. Protocols to detect senescence-associated beta-galactosidase (SA-betagal) activity, a biomarker of senescent cells in culture and in vivo. Nat Protoc. 2009; 4:1798-1806.
34. Joseph I, Tressler R, Bassett E, Harley C, Buseman CM, Pattamatta P, Wright WE, Shay JW and Go NF. The telomerase inhibitor imetelstat depletes cancer stem cells in breast and pancreatic cancer cell lines. Cancer Res. 70:9494-9504.

35. Goldblatt EM, Gentry ER, Fox MJ, Gryaznov SM, Shen $\mathrm{C}$ and Herbert BS. The telomerase template antagonist GRN163L alters MDA-MB-231 breast cancer cell morphology, inhibits growth, and augments the effects of paclitaxel. Mol Cancer Ther. 2009; 8:2027-2035. 